\title{
SPACES AND NETWORKS: CONCEPTS FOR SOCIAL STRATIFICATION
}

\author{
Joseph Woelfel and Monica Murero
}

\begin{abstract}
While early stratification students were deeply categorical in their theory and methods, concerning themselves with discrete categories or "bins" such as status, strata, classes, layers and such, Haller already conceived of stratification as a continuous, comparative process. While older theory held that each individual occupied a discrete status to which were attached role expectations which guided the individual's behavior, Haller conceived of status as a continuous hierarchy, through which individuals moved in a career trajectory over time.

The Galileo System of measuring social objects as points in a multidimensional Riemannian continuum is a deliberate effort to realize Haller's theoretical conception of a continuous array of statuses, occupations and positions through which individuals move in continuous arcs, impelled by the expectations of others and other forces.

In this paper we describe the fundamental premises of the Galileo model and present data showing the utility of the model.
\end{abstract}

The Shape of Social Inequality: Stratification and Ethnicity in Comparative Perspective

Research in Social Stratification and Mobility, Volume 22, 57-71

(C) 2005 Published by Elsevier Ltd.

ISSN: 0276-5624/doi:10.1016/S0276-5624(04)22002-9 


\section{CONCEPTS AND REFERENCE FRAMES}

Perhaps the great achievement of the social sciences in the 20th century was the discovery that reference frames and conceptual systems influence our perceptions of reality, and that these reference frames and conceptual systems are themselves socially constructed. Perhaps the great failure of the social sciences in the 20th century was the failure to develop formal technical criteria for evaluating reference frames and constructing "better" ones. Of course, reference frames and conceptual systems are routinely evaluated on political, moral and esthetic grounds, and activists commonly develop and implement new frames which benefit their cause (e.g. by insisting on terms such as "right to life" and "pro choice" instead of "anti-abortion" and "pro abortion"). Few social scientists, however, attempt to evaluate reference frames and conceptual systems from an information theoretic perspective, by questioning how well they serve as precise and efficient encoding systems.

With few exceptions, and in spite of frequent repetitions of words like "continuing" and "ongoing" in the works of theorists such as G. H. Mead (1934), even the most contemporary social theory and social research utilize the same conceptual system used by Aristotle: that is, a reference frame in which experience is "chopped up" into discrete categories, and in which behavior is described as discrete jumps from one categorical state to another. Moreover, contemporary social science continues to work within Aristotle's notion of entelechy, where each and every of these discrete jumps from one state to another is motivated by a goal or drive or need.

Before Galileo, the dominant model for understanding motion and change was Aristotle's entelechy. ${ }^{1}$ Within this philosophy, goal or intention is the primary mover of all things animal, vegetable and mineral. Fire rises because it seeks its proper place at the periphery of the world. Heavy objects fall because they are seeking their proper place at the center of the world. In the entelechy, water seeks its own level, nature abhors a vacuum, and everything seeks its proper place, each thing following goals in an unbroken chain established once and for all in the mind of the original "unmoved mover."

The physical sciences adhered to the Aristotelian categorical, teleological model until Galileo Galilei, who renounced the concept of goals for physical objects, and viewed physical motion and change instead as the response of matter to impartial "forces." Galileo also abandoned the categorical model of discrete jumps from one categorical state to another, and replaced it with a continuous model of motion based on ratios to an arbitrary standard unit of measure. 


\section{STATUS ATTAINMENT AS A COMMUNICATION PROCESS}

The social sciences, however, never passed through the scientific revolution, and the notion of goal is still preeminent as an explanation for human and social behavior. Indeed, the most common model of status attainment within sociology is one in which individuals are born into status which provide them with resources and impediments which facilitate or impede their goal-driven quest for achievement. However predominant such a view might be, it is possible to implement a model in which goals play a subordinate role, and indeed are themselves produced by impartial forces in the social and physical environment.

One particularly useful approach emphasizes the communication aspects of social structure and social processes. Viewed in this light, the social structure of any society consists in sets of definitions and beliefs of individuals about the way their lives are organized. A status, for example, can be defined as a set of expectations about the roles appropriate to its incumbents, as a role can be considered expectations about the behaviors appropriate to its holders. These definitions must be communicated to those who will occupy the status and execute the role as well as to those in other statuses and roles who will interact with those occupants. Indeed, every hundred years or so the entire population of the world dies, and must communicate the entire information structure that defines the society to another worldful of people.

A communication model, whatever the substantive context, focuses on the systems that encode, store, transmit and manipulate information. For stratification, communication research focuses on what are the expectations that define statuses and roles, how are these expectations encoded, where are they stored, and how are they communicated.

The theoretical model underlying the Wisconsin Significant Other Project ("Other than what?" you might ask) is largely a communication model. Based on what has been called "The Wisconsin Model" (Sewell et al., 1969), it assumes that individuals are born into statuses or locations in the society. These statuses present their incumbents with resources and encumbrances, and expose them to certain other people who observe their circumstances and communicate to them expectations for their activities. Of these "other people," some play a particularly important role in defining an individual's expectations, and these are called "significant others" in the model.

Based on the expectations which others communicate to them, along with their own self-reflexive observations of their situations and past actions, individuals form expectations about their own behaviors. These expectations, constrained by 
physical circumstances, genetic factors, good or ill fortune and the like, determine the activities of the individual. The word "expectation" is chosen here specifically to indicate that individuals behave in ways consistent with their understanding of who and what they are rather than in response to goals, needs, drives or other affective motivators (Lemert, 1950).

While this model is entirely consistent with an interactionist perspective, at the inception of the project there was almost no empirical data which addressed the effects of significant others on the attitudes, beliefs and expectations of individuals, particularly in a stratification context. The most significant data available were presented by Sewell et al. (1969) who showed that the likelihood that a high school student would choose to attend college was correlated with an index of three self-reported dichotomous measures: whether the student believed most of his/her friends, teachers and parents expected them to attend college.

Although a groundbreaking study of seminal importance, the Sewell et al. (1969) study was hampered by its underlying categorical conceptualization. All major concepts in the study are categorical: significant others' expectations are dichotomous (expect college, do not expect college), as are the students' own aspirations (expect to attend, don't expect to attend) and attainments (attend, not attend). ${ }^{2}$ Problems with this categorical model were understood particularly among the Wisconsin status attainment workers, and a major part of the motivation behind the Wisconsin Significant Other Project was to replace these categorical variables with numerical concepts.

Haller, who conceived and organized the Wisconsin Significant Other Project, understood that status could be viewed as a continuous variable, and worked to extend the range of status that could be precisely measured, particularly to very low levels in rural Brazil. He also developed the Occupational Aspiration Scale (OAS) based on occupational prestige scores from the NORC scale (Haller \& Miller, 1971). Haller also understood that status attainment was not a dichotomous, before/after measure, but rather each individual's status attainments represented a trajectory over time. The OAS measured short range and long range aspirations to tap the concept of the trajectory to some extent. Overall, the OAS rated adolescent's aspirations on an 80 point scale, considerably advanced over the dichotomous categorical measure in the Sewell et al. study (Woelfel \& Haller, 1971).

Based on the OAS, the Wisconsin Significant Other Battery (WISOB) measured both significant others' expectations and the adolescents' own aspirations on numerical scales. Moreover, the WISOB did not assume that certain categories of persons (e.g. parents, teachers, peer friends) were always significant, nor that they were the only significant persons. Rather, efforts were made to identify the exact significant others for each adolescent based on a simple theoretical model (Woelfel, 1967a, b). As a result, the Significant Other project replaced 
the three dichotomous significant others' expectations variables from the Sewell et al. (1969) study with a numerical level of each significant other's expectation for each significant other identified. These numerical expectation levels could be averaged across all significant others for each adolescent to yield a mean level of expectation.

These mean levels of significant others' expectations worked extremely well, explaining between half and two thirds of the variance in the adolescents' own numerical aspirations measured on identical scales, which was about double the variance explained in the Sewell et al. study (Woelfel \& Haller, 1971).

While the average expectations were initially calculated as a heuristic device to deal with the variable number of significant others per case, subsequent analysis showed that the procedure made theoretical sense, since the mean represented that point at which all the "forces" expressed by the expectations of the significant others would balance so that the net force acting on the adolescent would be zero (Woelfel \& Hernandez, 1973). The underlying simple theory suggests that each significant other's expectation can be represented as a force vector pulling the adolescent in a specific direction, and that, over time, the individual's own attitude will tend toward the point at which all such forces are balanced.

Several studies showed that this theory worked quite well in several other contexts in addition to status aspirations, including political radicalism (Gillham \& McPhail, 1974), cigarette smoking (Mettlin, 1973), jury decisions (Mistretta et al., 1973), and others (e.g. Danes et al., 1984; Saltiel \& Woelfel, 1975). Within the area of status attainment, empirical support for the model has been consistently strong (Lin et al., 1981; Picou \& Campbell, 1975; Saltiel, 1975, 1978, 1983).

\section{THE GALILEO MODEL}

A fundamental aspect of the "linear force aggregation" theory implied by the averaging model is the inherent interdependence of the theoretical calculus and its measurement system. The idea that an individual's attitudes and beliefs tend toward the mean of the information received pertinent to those attitudes and beliefs assumes directly that attitudes, beliefs and the information environment can all be measured on continuous numerical scales. At the time, however, (and to a considerable extent even today) most social scientists believed that variables, by their inherent nature, were measurable only at certain levels, i.e. ratio, interval, ordinal or nominal. The averaging theory could be expected to work only for the first two of these types, leaving important stratification variables, such as occupational choice, outside the theory. If a child's mother, for example, wants her to finish college (16 years of schooling) and her father expects her finish high 
school (12 years), the average is clearly 14 . But what if her mother expects her to become an accountant and her father expects her to become a stenographer? What is the average of "accountant" and "stenographer?" Within the "force aggregation theory" the mean of accountant and stenographer is undefined.

Meanwhile, Woelfel (1980a) and his students at the University of Illinois had developed procedures for representing discrete "objects" in a continuous spatial array. Usually referred to as the "Galileo" 3 model, it takes as its elementary unit the "object," which, following Blumer, is "anything that can be designated or referred to..." (Blumer, 1966) and assumes that the perceived dissimilarity between any two "objects" can be measured on a continuous scale.

The original scale used by Woelfel and his students was a ratio paired comparisons design, where respondents were given a "criterion pair": (e.g. "If 'Bank Teller' and 'Postal clerk' are 100 units apart..."), then asked to estimate the differences among all possible pairs of the other $n$ objects scaled: (" $\ldots$ how far apart are $\mathrm{a}$ and $\mathrm{b}, \mathrm{a}$ and $\mathrm{c}, \ldots \mathrm{a}$ and $\mathrm{n}, \ldots n-1$ and $n$ ).

Although an unusual format for scaling at the time, the complete paired comparison ratio estimation task is generally considered the single most precise form of measurement known to psychometricians, and substantial evidence in the case specifically of Galileo scales indicates that they can attain considerable precision even at small sample sizes (Barnett, 1972; Gillham \& Woelfel, 1977; Gordon, 1976; Gordon \& DeLeo, 1975; Woelfel et al., 1980).

This procedure was applied to the area of occupational attainment by Saltiel (1983). Using a device based on the Occupational Aspiration Scale (Haller \& Miller, 1971), he identified the 34 most frequently chosen occupations for high school students in a consolidated rural school district in Montana. The perceived dissimilarities among these occupations were measured using a ratio-scaled pair comparison Galileo scale; $1 / 3$ of all possible pairs chosen at random were estimated by each student and by each of the student's significant others, who were identified by the Wisconsin Significant Other Battery.

These measurements produced a multidimensional space, averaged across all students and their significant others, within which are arrayed the 34 occupations.

Figure 1 represents a subset of 13 of the 34 Saltiel occupations. Earlier onedimensional arrays of occupations can be related to this figure easily. The Duncan Socioeconomic Index (SEI) correlates about 0.9 (corresponding to an angle of about 26 degrees) with the first principle (left-right) axis of this space, for example.

Position in this space has no absolute significance, but relative position is important. Occupations arrayed close to each other are perceived to be similar by respondents, while those far apart are perceived to be different in proportion to their distance apart. Most important, since each occupation's position in the space is given by a vector of numerical coordinates, it is possible to take the average of 


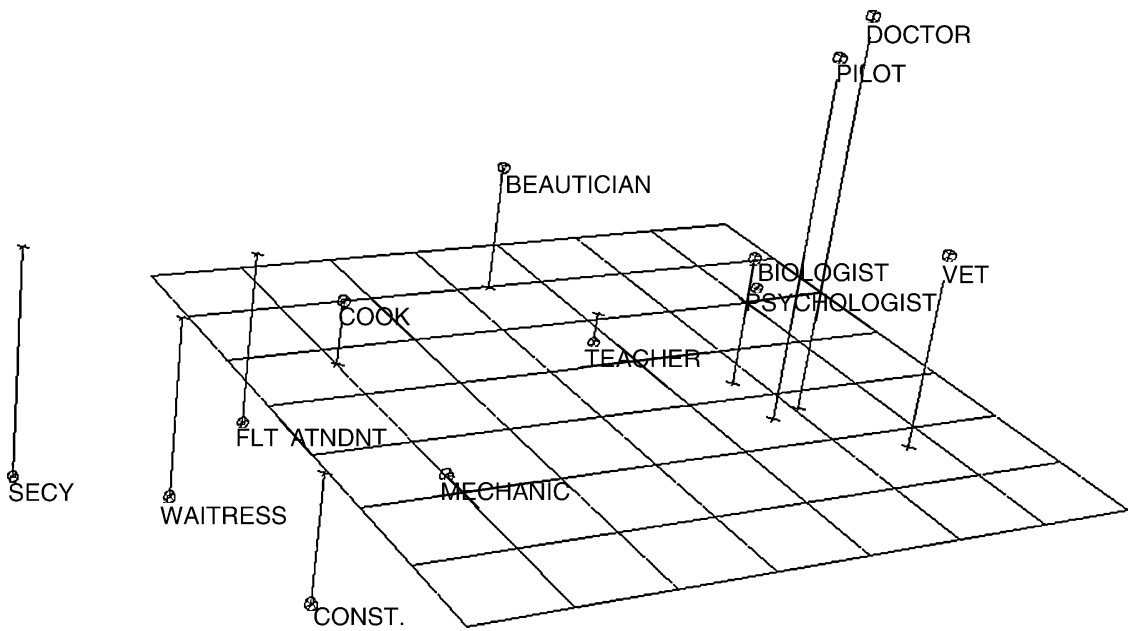

Fig. 1. View of a Subset of Saltiel's 34 Occupations.

2 or more occupations by taking the average of their coordinates. The results will be the coordinates of a point in the space.

This makes it possible to generalize the equations of the "linear force aggregation theory" to discrete choice situations in general and to occupational choice in particular. Simply put, the general theory suggests that the occupation chosen by any given student will lie close to the point defined by the average of the coordinates of the specific occupations expected for the child by its significant others. ${ }^{4}$ Indeed, a variant of the Woelfel and Haller regression model, augmented by the mean coordinates of the significant others' discrete expectations, does even better in the choice situation than the original educational and occupational aspiration model, accounting for $84 \%$ of the variance in the average job choice on the first dimension, $93 \%$ of the variance on the second, and averages about $60 \%$ explained variance on the remaining (much smaller) dimensions.

The Galileo Model not only makes it possible to generalize the Wisconsin Status Attainment Model to the case of discrete occupational choice, but it provides a particularly favorable frame of reference for visualizing Haller's notion of a lifetime status trajectory. To do this, it is necessary to generate a sequence of such spaces over time, provide a common orientation, and then simply note the trajectory through the series of occupations occupied by any individual over time.

The development of a sequence of spaces is straightforward, and simply requires repeated measurements over time. Providing a common orientation, however, is not so straightforward, since occupational space, like physical space, has no 
"privileged" orientation. It is not given by nature, for example, that North should appear at the top of all maps, and East to the right, with altitude represented as a perpendicular to the north-south east-west plane. These are conventions arrived at by negotiation and dispute over several hundreds of years.

Choices of these reference standards have important consequences for the dynamic characteristics of the trajectories of objects. Consider, for example, the consequences of defining the earth rather than the sun as the center of the solar system, perhaps the most famous case of an unwise choice of reference frame in human history. In such a system, huge unbalanced masses revolve around a miniscule mass itself located quite far from the center of mass of the entire system, resulting in equations of motion that are very complicated and which require either that we believe in magic (the solar system does not obey physical law) or huge unexplained forces. What's more, the motions observers will actually see in such a system will be peculiar, since planets will occasionally seem to reverse their motion for no apparent reason. Objects will appear to behave capriciously, leading serious observers to conclude that phenomena are in fact not law governed, or, at the least, too complicated to be understood in a naturalistic scheme.

The mathematical solution for aligning reference systems (known as a Galilean transformation after Galileo) has been known since Galileo for three dimensional Euclidean spaces, but a general mathematical solution and computer algorithm for high dimensional non Euclidean spaces was developed by Woelfel and his coworkers (Woelfel, 1980b; Woelfel \& Barnett, 1992; Woelfel \& Fink, 1980; Woelfel et al., 1976, 1979, 1986, 1989). The only transformations allowed are "rigid body transformations," that is, spaces may be rotated and/or translated in any arbitrary way, so long as the interpoint distances in any space are not altered. If a twodimensional space were drawn on a piece of paper, for example, the paper could be moved, turned upside down, reflected in a mirror, put top to bottom, or any such motion, but it could not be folded or bent.

Woelfel and Barnett (1992) illustrate the principles underlying the choice of a reference frame with a clock viewed over several intervals of time. Under normal circumstances, clocks are affixed to walls, set atop furniture or otherwise arrayed against objects we believe to be stable and unmoving. As time passes, the distances among the hands and the numbers on the clock face change, as do the distances among the clock hands and everything else in the reference frame. But if we consider the clock in otherwise empty space it will not be evident as time passes whether the hands are moving relative to the numbers, the numbers relative to the hands, or some combination of both. Indeed, any of these solutions is as good as any other, but only one of them is the simplest to perceive and remember: that is, the solution in which the numbers and pivot point remain fixed and only the hands move. $^{5}$ 
The clock numbers and pivot provide fixed reference points against which the motion of the hands can be gauged. In the case of the clock example, these fixed reference points are well known, and can be specified as constraints on the rotation; in words, the constraints mean "rotate and translate each of the spaces in this time sequence until the motion of these fixed points is minimized."

In less familiar areas, such may not be the case. Woelfel et al. (1989), considered the case of people's conception of the days of the week, themselves and certain kinds of activity. 414 randomly selected telephone subscribers in the Albany, NY area were asked to respond to a Galileo-type paired comparison instrument assessing their perceptions of the differences among the seven days of the week (Sunday through Saturday), themselves, and activities such as work, recreation, and the like. Data were collected for each of 23 days.

The authors tried several rotations schemes, but found one in which the days and activities were held as close to motionless as possible and the respondent's self point was allowed to move freely. In this model, the days of the week form a roughly elliptical figure, the recreational activities are closest to the weekend, while "work" is located closest to Monday. As days pass, the self point orbits around the ellipse formed by the days, being closest to Sunday on Sunday, Monday on Monday, etc. As the self approaches the weekend, it grows closer to the recreational activities, and, as it moves closer to Monday, it moves correspondingly closer to work.

\section{LAWS OF MOTION AND CHANGE}

Woelfel and Barnett generalize beyond establishing fixed reference points to constrain reference frame selection, and suggest the possibility of constraining the transformation algorithm to produce a reference frame in which a given set of "laws of motion" will hold. ${ }^{6}$ Kincaid et al. (1981) used the Galileo model to measure the beliefs of Korean immigrants to Hawaii, and that of the general Hawaiian public. They showed that the cultural space of the Koreans converged on the general Hawaiian cultural space as a function of the length of time they had lived in Hawaii, and further that the rate of convergence fit the generalized Newtonian equation for convergence of equilibrium thermodynamic systems to within measurement error. Becker (1993) showed that the convergence of Brazilian Japanese into Japan followed a similar model.

Woelfel et al. (1986) showed a randomly generated paragraph describing six imaginary people to 75 undergraduates at the University at Albany, then had each of them fill out a Galileo type instrument assessing the perceived differences among the six people after a randomly chosen waiting period of between one and 178 hours. Results of an earlier pretest had shown the space increased in size across 
the first hour; these results showed the size of the space exhibited a substantial rise at 9 hours, falling back to baseline at around 14 hours. An exact replication a year later, this time with 557 students participating, yielded very similar results.

Foldy and Woelfel (1985) showed in a similar experiment, with one hour time intervals, that the spaces of people who heard persuasive messages not only grew after reading the message, but exhibited oscillations consistent with a perturbed equilibrium dynamic system. This work is itself consistent with independent research by Fink and Kaplowitz (1993) and Kaplowitz and Fink (1996, 1997) showing that, even at very small and precisely measured time intervals, observed attitude changes are well modeled as damped harmonic oscillating systems.

\section{NEURAL NETWORKS AS A BASIS FOR GALILEO SPACES}

Recent work in neuroscience, computer science, psychology, communication and other areas has had an important impact on our understanding of cognitive processes. Somewhat simplified, neurons are cells which can be "activated" by stimulation. When photons fall on the retina of an eye, for example, they stimulate receptor cells which transmit electrochemical energy through the optic nerve to a series of neurons. Different visual patterns on the retina will lead to different patterns of activation among the neurons; these patterns of activation of neurons represent the brain's mechanism for representing external images internally.

When the photons stop impacting the retinal receptor cells, the neurons deactivate, and the internal representation of the pattern is lost. But if the same pattern is repeated with some frequency, the neurons which collectively represent the pattern tend to become connected to each other. When these connections are of sufficient strength, the pattern is remembered. Since the cells are interconnected, activation of a sufficient subset of them will result in activation of the rest via the interconnections.

This collection of perceptual elements into an interconnected pattern represents a solid physiological basis for Mead's concept of an "object." Moreover, each such cluster can be interconnected with still other clusters, and these, in turn can be connected with still others. ${ }^{7}$ Each of these "objects" is defined entirely in terms of its relationship to the other concepts of which individuals are aware. Not only is this model consistent with Mead's understanding, but it is the foundation of the Galileo model as well (Woelfel, 1993a, 1997; Woelfel et al., 1993).

The most widely known technology based on the integration of neural models with the Galileo model is CATPAC ${ }^{\odot}$. Because of its underlying neural technology, 
CATPAC (CATegory PACkage) is able to serve as a bridge between categorical conceptualizations and the continuous Galileo representation (Woelfel, 1993b).

The logic of CATPAC is identical to the logic of situated meaning in Mead. In CATPAC, a moving window (default size is 7 words) sweeps through a text. If we consider the window to be a "situation," then, initially, the first seven words in the text will be in the situation. Each of them is represented by an artificial neuron, which is activated when the word is in the situation (window). Connections among all active neurons are then incremented by a small amount. The window then slides one word to the right, and generates a second "situation." Once again, those neurons representing the words found in the new situation are activated, connections among all active neurons are incremented, and the window slides again.

At each "cycle" of this system, all connections among all neurons are slightly decremented to simulate forgetting. As a result, connections that happen infrequently will be lost, but those which co-occur with more frequency will be strengthened, so that words which often are found in the same situation will be tightly connected. The situated meaning of each word is given by its relationships to the other words in that situation. Thus CATPAC will define "Mustang" one way when situated in a context which includes Morgan, Palomino, etc., but another way in a context which includes Camero, Challenger, Firebird, and the like.

The Galileo model underlying CATPAC is completely general, and applies to any kind of objects found in any kind of situation. The program Oresme, for example, reads lists of objects found in any "bunch," such as items in a grocery cart, foods eaten at a given meal, movies favored by a person, and learns to relate them to each other following the procedures described above. The result is situated definitions of objects based on their interrelationship to other objects within situations. As with CATPAC, the interrelationships among the objects can be taken as the basis for plotting those objects in Galileo space, such that the more similar objects will be near each other, and different objects further apart.

\section{CURRENT RESEARCH}

The Galileo model and its newer associated neural model have developed widespread applications, and are used around the world in the public and private sector wherever procedures to influence attitudes, beliefs and behavior are used. Typical uses include worldwide studies of attitudes toward rain forest use in developing nations, land, park, logging, fishing, hunting and other uses among stakeholders in the Yukon, election campaigns, commercial advertising and market research, and academic research. 
One of the more interesting areas of pure research focuses on the core notion in the Galileo model that meaning lies in the interrelationship among elements in a system, rather than in the elements themselves. This provides a renewed interest in the notion of the Collective Consciousness, particularly as the explosive development of the Internet expands the network of connections among people and groups worldwide. Langhorne (2000), for example, has made maps of individual's and groups perception of the Internet by reading the co-occurrences of site visits during Internet sessions into CATPAC; the resulting maps show websites whose distances apart are based on the actual usage by people. These "meanings" exist not only within the minds of individuals, but also in the patterns of interconnections among the individuals - the "collective" has knowledge and meanings which are unknown to the constituent individuals.

Woelfel (2000) has read descriptions of scenes to large assemblies of individuals, who are then asked to estimate the distances among the objects in the scenes. They are also asked to draw the scenes. Evidence so far from several hundred individuals indicates that virtually none of them can draw the scenes accurately after hearing the descriptions. The average of the distance estimates of all the individuals, however, reproduces the scenes with considerable precision; random split halves match each other; but systematic differences appear when, for example, the average of all males is compared to the average of all females. This provides suggestive evidence that the collection of people knows something that none of its constituents know, and that that knowledge can be measured by Galileo procedures.

There is as yet no specific theory available to indicate whether the collective consciousness has any capacity to develop self reflexivity, although the increasing connectedness of the Internet and the extensive focus of attention on its development resemble in general the kinds of communication mechanisms which develop self awareness in individuals. Moreover, an exponential increase in the technology of communication and display might at least in theory generate the kind of virtual experience that might parallel Durkheim's totemistic ritual on a much large scale. The consequences of this explosive development of the interconnectedness of people and organizations, along with an understanding that collective concepts consist of patterns in the matrix of interconnections brings on many fascinating opportunities for theory and research in the Galileo model.

\section{NOTES}

1. Aristotle was left to dominate human understanding of motion and change because his great rival Plato believed that motion and change were illusions not worthy of study. 
2. To be sure, Sewell, a strong advocate of precise measurement of stratification variables, was well aware of these difficulties, but the data used for the analysis were collected by another investigator for another purpose.

3 . These procedures have been described by a variety of names over the years, including multidimensional scaling, metric multidimensional scaling, and others, but these nomenclatures conflict with standard usage in psychometrics and mathematics. The only precise, unambiguous term in use is probably The Galileo Model, or, equivalently, The Galileo System.

4. Each significant other's expectation will be represented by a point in the occupation space. The set of all expectations for each student will form a geometric figure, e.g. any two will form a line segment, any three a triangle, any for a quadrilateral, and so on. The average of the coordinates will describe the exact geometric center of that figure. As in the Force Aggregation model, choosing an occupation near this point will minimize the total discrepancy between the student's choice and the set of all significant other expectations.

5. If this does not seem obvious, consider how much more difficult it would be to tell the time in a single glance if the clock face rotated as well as the hands.

6. It is important to realize that, while it is possible to generate an infinite number of reference frames by this method, it is not possible to generate reference frames which will produce any arbitrary outcome.

7. When represented mathematically, these interconnections can be seen to be a matrix of "connection strengths." This matrix can be seen as a kind of "similarities matrix" because neurons that are very frequently active in the same patterns will be tightly connected, and represented by a high numerical value. "Centering" this matrix, i.e. expressing it as deviation scores from the mean connection strength, will cause similar nodes to be represented by relatively large positive values, and dissimilar nodes to be represented by relatively large negative numbers. This similarities matrix (technically a scalar products matrix) is formally equivalent to the centroid scalar products matrix from which Galileo spaces are calculated, and serve as the basis for Galileo mappings.

\section{REFERENCES}

Barnett, G. A. (1972). Reliability and metric multidimensional scaling. East Lansing: Department of Communication, Michigan State University.

Becker C. B. (1993). A communication based theory of cultural reproduction and transformation. Information and attitude change among Brazilian immigrants in Japan. Ph.D. Dissertation. Presented to the State University of New York at Buffalo, Department of Communication.

Blumer, H. (1966). Commentary and debate. American Journal of Sociology, 71, 535-547.

Danes, J., Hunter, J. E., \& Woelfel, J. (1984). Belief change and accumulated information. In: J. E. Hunter, J. E. Danes \& H. S. Cohen (Eds), Mathematical Model of Attitude Change (Human Communication Research Series, Vol. 1). Orlando, FL: Academic Press.

Fink, E. L., \& Kaplowitz, S. A. (1993). Oscillation in beliefs and cognitive networks. In: G. A. Barnett \& W. D. Richards (Eds), Progress in Communication Science (pp. 247-272). Norwood, NJ: Ablex Publishing Corporation.

Foldy, J., \& Woelfel, J. (1985). Conceptual structures as damped harmonic oscillators. Paper presented to the International Communication Association, Honolulu. 
Gillham, J. R., \& McPhail, T. L. (1974). Political radicalization as a communication process. Communication Research, 1, 243-263.

Gillham, J. R., \& Woelfel, J. (1977). The Galileo system of measurement: Preliminary evidence for precision, stability, and equivalence to traditional measures. Human Communication Research, 3(3), 243-263.

Gordon, T. F. (1976). Subject abilities to use MDS: Effects of varying the criterion pair. Paper presented to the Association for Education in Journalism, College Park, MD.

Gordon, T. F., \& DeLeo, H. C. (1975). Structural variations in 'Galileo' space: Effects of varying the criterion pair in metric multidimensional scaling. Unpublished Manuscript. Philadelpha: Temple University.

Haller, A. O., \& Miller, I. W. (1971). The occupational aspiration scale: Theory, structure and correlates. New York: Shenkman.

Kaplowitz, S. A., \& Fink, E. L. (1996). Cybernetics of attitudes and decisions. In: J. Watt \& C. A. Van Lear (Eds), Dynamic Patterns in Communication Research (pp. 277-300). Newbury Park, CA: Sage.

Kaplowitz, S. A., \& Fink, E. L. (1997). Message discrepancy and persuasion. In: G. A. Barnett \& W. D. Richards (Eds), Progress in Communication Science (Vol. 12). Norwood, NJ: Ablex Publishing Corporation.

Kincaid, D. L., Yum, J. O., Woelfel, J. \& Barnett, G. A. (1981). The cultural convergence of Korean immigrants in Hawaii: An empirical test of a mathematical theory. Paper presented to the International Communication Association, Minneapolis.

Langhorne, A. (2000). Mapping the internet: Using neural networks to develop graphical internet navigation profiles in organizations. Doctoral dissertation. State University of New York at Buffalo.

Lemert, E. (1950). Social pathology. New York: McGraw-Hill.

Lin, N., Ensel, W. M., \& Vaughn, J. C. (1981). Social resources and strength of ties: Structural factors in occupational status attainment. American Sociological Review, 46(4), 393-405.

Mead, G. H. (1934). Mind, self and society. Chicago: University of Chicago Press.

Mettlin, C. (1973). Smokin as behavior: Applying social psychological theory. Journal of Health and Social Behavior, 14, 145-152.

Mistretta, M., Miles, W., \& Barnett, G. A. (1973). Jury and judicial decision-making: A research design and preliminary report. Paper presented to the American Sociological Association, New York.

Picou, S. J., \& Campbell, R. E. (1975). Career behavior of special groups. Columbus: Merrill Publishing Company.

Saltiel, J. M. (1978). Predicting occupational choice: A multidimensional scaling approach. Paper presented at the First Annual "Metric Multidimensional Scaling Workshop". International Communication Association, Chicago.

Saltiel, J. M. (1983). An application of the Wisconsin model of status attainment to the occupational choice process. Unpublished manuscript. Department of Sociology, Montana State University, Bozeman, MT

Saltiel, J. M., \& Woelfel, J. D. (1975). Inertia in cognitive processes: The role of accumulated information in attitude change. Human Communication Research, 1, 333-334.

Sewell, W. H., Haller, A. O., \& Portes, A. (1969). The educational and early occupational status attainment process. American Sociology Review, 34, 82-92.

Woelfel, J. D. (1967a). A paradigm for research on significant others. Unpublished Working Paper. Madison: University of Wisconsin. 
Woelfel, J. D. (1967b). Others' expectations and their effects on the attitudes of an individual. Unpublished Working Paper. Madison: University of Wisconsin.

Woelfel, J. D. (1980a). Foundations of cognitive theory. In: D. P. Cushman \& R. Mcphee (Eds), Explorations in the Message Attitude Behavior Relationship. New York: Academic Press.

Woelfel, J. D. (1980b). Variational principles of communication. Paper presented to the International Communication Association, Acapulco, Mexico.

Woelfel, J. D. (1993a). Cognitive processes and communication networks: A general theory. In: G. A. Barnett \& W. D. Richards (Eds), Progress in Communication Science (Vol. 12). Norwood, NJ: Ablex Publishing Corporation.

Woelfel, J. D. (1993b). Artificial neural networks in policy research: A current assessment. Journal of Communication, 43(1), 63-80.

Woelfel, J. D. (1997). Attitudes as nonhierarchical clusters in neural networks. In: G. A. Barnett \& W. D. Richards (Eds), Progress in Communication Science (Vol. 12). Norwood, NJ: Ablex Publishing Corporation.

Woelfel, J. D. (2000). Collective representation and distributed encoding. In progress paper. State University of New York at Buffalo.

Woelfel, J. D., \& Barnett, G. A. (1992). Procedures for the comparison of time dependent data to a theoretical criterion. Quality and Quantity: A European Journal of Methodology, 26, 367-381.

Woelfel, J. D., Barnett, G. A., Pruzek, R., \& Zimmelman, R. (1989). Rotation to simple processes: The effect of alternative rotation rules on observed patterns in time-ordered measurement. Quality and Quantity, 23, 3-20.

Woelfel, J. D., \& Fink, E. L. (1980). The measurement of communication processes: Galileo theory and method. New York: Academic Press.

Woelfel, J. D., \& Haller, A. O. (1971). Significant others, the self-reflexive act, and the attitude formation process. American Sociological Review, 36(1), 74-87.

Woelfel, J. D., \& Hernandez, D. (1973). A theory of linear force aggregation. Unpublished monograph. Champaign: University of Illinois.

Woelfel, J. D., Holmes, R. A., Cody, M., \& Fink, E. L. (1976). Message strategies in Riemman space. Paper presented before Joint Session of the Psychometric Society and the Mathematical Psychology Group, Durham, NC.

Woelfel, J., Holmes, R., Kincaid, D. L., \& Barnett, G. A. (1980). How to do a Galileo study. Troy, NY: Good Books.

Woelfel, J. D., Newton, B., Holmes, R., Kincaid, D. L., \& Lee, J. (1986). Effects of compound messages on global characteristics of Galileo spaces. Quality and Quantity, 20, 133-145.

Woelfel, J. D., Richards, W. D., Jr., \& Stoyanoff, N. J. (1993). Conversational networks. In: G. A. Barnett \& W. D. Richards (Eds), Progress in Communication Science (Vol. 12). Norwood, NJ: Ablex Publishing Corporation. 